\title{
Testing planet formation theories with giant stars
}

\author{
Luca Pasquini ${ }^{1}$, M.P. Döllinger ${ }^{1}$, A. Hatzes ${ }^{2}$, J. Setiawan ${ }^{3}$, \\ L. Girardi ${ }^{4}$, L. da Silva ${ }^{5}$, J.R. de Medeiros ${ }^{6}$ and A. Weiss ${ }^{7}$ \\ ${ }^{1} \mathrm{ESO}$ \\ Garching, Germany \\ email: lpasquin@eso.org, mdoellin@eso.org \\ ${ }^{2}$ Thüringer Landessternwarte \\ Tautemburg, Germany \\ email: artie@tls-tautenburg.de \\ ${ }^{3} \mathrm{MPiA}$ \\ Heidelberg, Germany \\ email: setiawan@mpia-hd.mpg.de \\ ${ }^{4}$ INAF-OaPD \\ Padova, Italy \\ email: leo.girardi@oapd.inaf .it \\ ${ }^{5} \mathrm{ON}$ \\ Rio de Janeiro, Brazil \\ email: licio@on.br \\ ${ }^{6} \mathrm{UFRN}$ \\ Natal, Brazil \\ email: renan@dfte.ufrn.br \\ ${ }^{7} \mathrm{MPA}$ \\ Garching , Germany \\ email: weiss@mpa-garching.mpg.de
}

\begin{abstract}
Planet searches around evolved giant stars are bringing new insights to planet formation theories by virtue of the broader stellar mass range of the host stars compared to the solar-type stars that have been the subject of most current planet searches programs. These searches among giant stars are producing extremely interesting results. Contrary to main sequence stars planet-hosting giants do not show a tendency of being more metal rich. Even if limited, the statistics also suggest a higher frequency of giant planets (at least $10 \%$ ) that are more massive compared to solar-type main sequence stars.

The interpretation of these results is not straightforward. We propose that the lack of a metallicity-planet connection among giant stars is due to pollution of the star while on the main sequence, followed by dillution during the giant phase. We also suggest that the higher mass and frequency of the planets are due to the higher stellar mass. Even if these results do not favor a specific formation scenario, they suggest that planetary formation might be more complex than what has been proposed so far, perhaps with two mechanisms at work and one or the other dominating according to the stellar mass. We finally stress as the detailed study of the host stars and of the parent sample is essential to derive firm conclusions.
\end{abstract}

Keywords. planetary systems: formation, stars: abundance, stars: fundamental parameters.

\section{Introduction}

Out of the more than 200 exoplanets known, only an handful orbit around evolved giants stars. These stars, however, are interesting targets for planet searches. Unlike 
the host stars of most known exoplanets which have a mass distribution that peaks at $0.8 \pm 0.3$ solar masses, giant stars can have masses several times this value. The radial velocity (RV) method, the most succesful technique at finding exoplanets, is insentive to more massive, early-type stars. These hot stars have few spectral lines that are often broadened by high stellar rotation rates. The typical RV error for a hot early-type star might be $100-1000 \mathrm{~ms}^{-1}$, much higher than the $\approx 10 \mathrm{~ms}^{-1}$ needed to detect sub-stellar companions. On the other hand, early-type main sequence stars that have evolved off the main sequence and have become giants have a plethora of narrow asorption lines that are amenable to RV planet searches. Figure 1 clearly shows this difference, where the spectral region of a main sequence A star is compared to the same spectral region of a giant star with approximately the same mass. While in the main sequence star only one broad feature is visible, in the giants many, narrow lines are present.

- The first interest in searching planets around giants is therefore to enlarge the stellar mass range surveyed, including higher masses.

Giants differ from main sequence stars in their structure: they have much larger radii (typical of $10 \mathrm{R}_{\odot}$, see (da Silva et al. 2006) and they have a much deeper convective zone compared to solar-type stars (cfr. discussion later). Differences in stellar radius and depth of the convection zone are expected also along the main sequence, but their variations are small compared to the large differences occurring when the stars evolved into giants. So it shall be much more evident and easy to detect those effects, such chemical pollution, whose signatures vary with internal structure changes.

- The second interest in searching planets around giants is therefore that, thanks to their different structures, giants provide ideal testbed to understand which stellar or planetary system characteristics depend from some stellar parameters, such as depth (mass) of the convective zone or stellar radius, which change radically when passing from the main sequence to the giant phase.

Interestingly, giants have been early recognized as radial velocity variable, and they could be broadly characterized by two variability timescales:

(a) Short timescales, of the order of a few days or fraction of day. These are most likely due to pulsations or solar-type oscillations (Hatzes \& Cochran 1994, Frandsen et al. 2002)

(b) Long timescales, of the order of one hundred or a few hundred days, either due to planetary companions or to stellar surface structure. (Hatzes \& Cochran 1993).

Because giant stars have higher intrinsic "noise" due to stellar oscillations, these stars have been so far neglected by planet searches. Although the situation is rapidly evolving, as testified by the contributions to this conference (Quirrenbach; Liu; Masasshi, reports in IAUS249 2007; Niedzielski 2008). In addition, one program is dedicated at Lick Observatory to follow up evolved A stars (Johnson et al. 2007a).

\section{The FEROS and the Tautenburg Surveys}

Our group started to obtain accurate radial velocity of giant stars in 1999, with the thesis work of J. Setiawan (Setiawan et al. 2003b, 2004 ) who used the FEROS spectrograph at the ESO 1.5m telescope. Seventy-six stars were observed for four years, and the most promising were followed up in the last years after the spectrograph was moved at the $2.2 \mathrm{~m}$ telescope, using time allocated to the Max Planck Institute for Astronomy. We will refer in the following to this sample as the 'southern' sample.

In 2004 M. Döllinger started a survey with the $2 \mathrm{~m}$ Alfred Jensch telescope in Tautenburg on 67 stars in the northern sky. 
In both cases the selected stars are giants (class III) with accurate Hipparcos parallaxes, known to better than $10 \%$. For the Tautenburg sample we added the constraint that the stars should be circumpolar so that observations could be obtained throughout the year. Intrinsically bright and thus cool giants were not selected for our samples to avoid the possible occurance of pulsating AGB stars which may have large RV amplitude variations and the fact that it is difficult to characterize their stellar parameters (cfr. next sections).

Figure 2 shows the colour-magnitude diagram of all our sample stars. Clearly we sample well the RGB, the clump and the beginning of the AGB. Very few subgiants were included.

\section{Characterization of the Stars}

Shortly after the first exoplanet has been discovered (Mayor and Queloz 1995) it was clear that it is essential to characterize the host stars. Age, mass and metallicity are fundamental parameters to understand the planetary formation mechanism and the relationship between stars and protoplanetary disks. The early discovery of the 'planetmetallicity' connection (Gonzalez 1997, Santos et al. 2000, 2001) was exciting and triggered more observational studies and theoretical efforts. When dealing with giants, the characterization of the stars is particularly important and also more difficult because of the presence of two degeneracies. First, while stars of different masses are quite well separated on the main sequence, while ascending the RGB (and in the clump) they occupy more or less the same region of the color-magnitude diagram. Second, evolutionary tracks of metal poor giants are bluer than for the tracks of metal rich giants. Thus an old metal poor star will occupy the same region of the CMD as a younger metal rich giant. This is the well known age-metallicity degeneracy. Thus to fully characterize a giant star (mass,

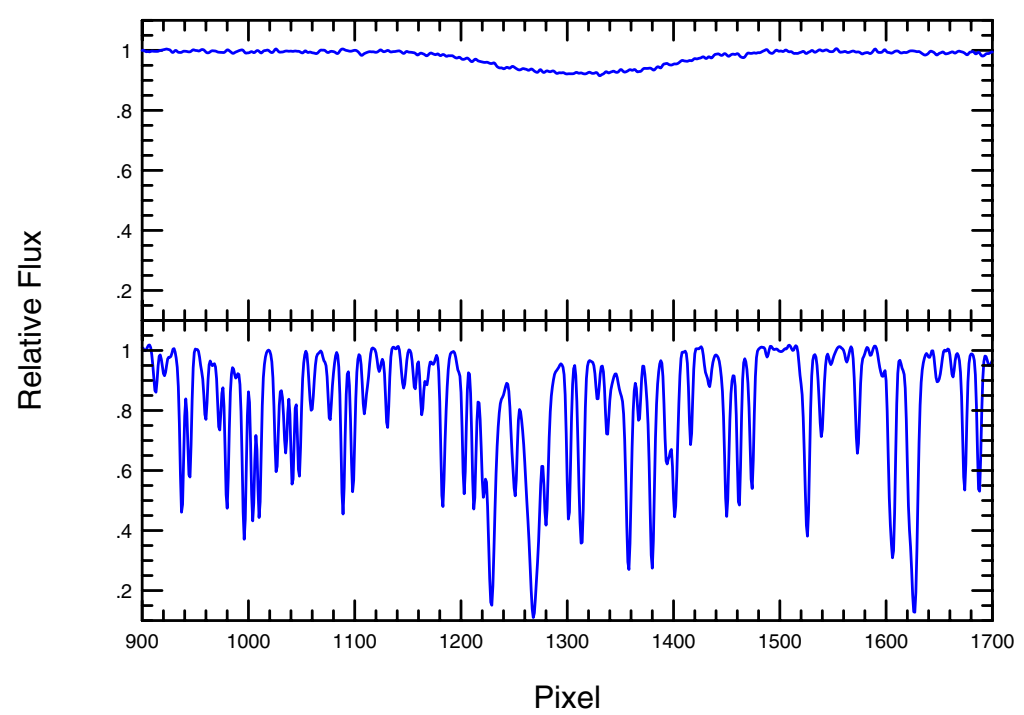

Figure 1. Portion of spectrum in the optical region of two stars: one massive main sequence star and one cool giant. This comparison shows that as soon as the star evolves from the main sequence migrating in the giant domain, it develops a very crowded line system, suitable for precise Radial Velocity (RV) measurements. 


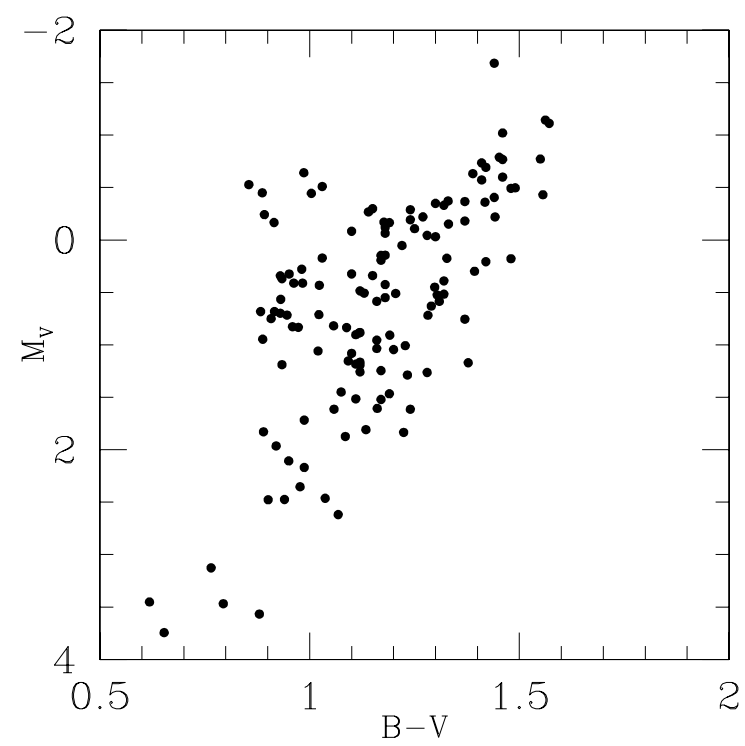

Figure 2. Color-magnitude diagram of the sample stars, observed with FEROS and at Tautenburg. The stars well sample the Red Giant Branch (RGB), the clump and the beginning of the AGB. Only a few subgiants are present, and a few cool, luminous stars.

radius, etc.) using evolutionary tracks requires the analysis of high resolution spectra from which effective temperature and metallicity are derived.

Effective temperature and metallicity were derived using a classical abundance analysis in a plane parallel atmospheres and LTE conditions. From these values the age and mass have been derived using a modified version of the Jorgensen and Lindegren (2005) method which computes the probability distribution function (PDF) of each quantity using theoretical tracks (cfr. da Silva et al. 2006 ) The exact shape of the PDF differs from star to star because that depends on the parameters errors and mostly on the position of the star in the CMD. In most instances the PDFs are sharply peaked and thus result in a well-determined parameter (Figure 3). Some stars, however, can have more uncertain parameters due to a broader, or even double-peaked PDF. da Silva et al. (2006) have performed several sanity checks on the recovered stellar parameters by comparing the derived colors and radii with observed ones. They found excellent agreement with the values derived with our PDF method.

We have further measured the effective temperatures of all the stars of the southern sample using the line-depth ratio method (Gray 1991, Biazzo et al. 2007a). They agreed very well with the temperatures derived with the abundance analysis. There was a small zero point difference $(5 \mathrm{~K})$ and a dispersion of less $70 \mathrm{~K}$, as shown in Figure 4. Finally, applying the same PDF method to six giants of the open cluster IC4651 we obtain for the stars of this cluster a mass of $2.0 \pm 0.2$ solar mass and an age of $1.2 \pm 0.2$ Gyrs, very similar to what is quoted in the WEBDA database (Biazzo et al. 2007b).

We are confident that we are able to produce reliable parameters for our sample stars, and we have applied the same analysis also to the Northern sample (Döllinger et al. 2008a). 


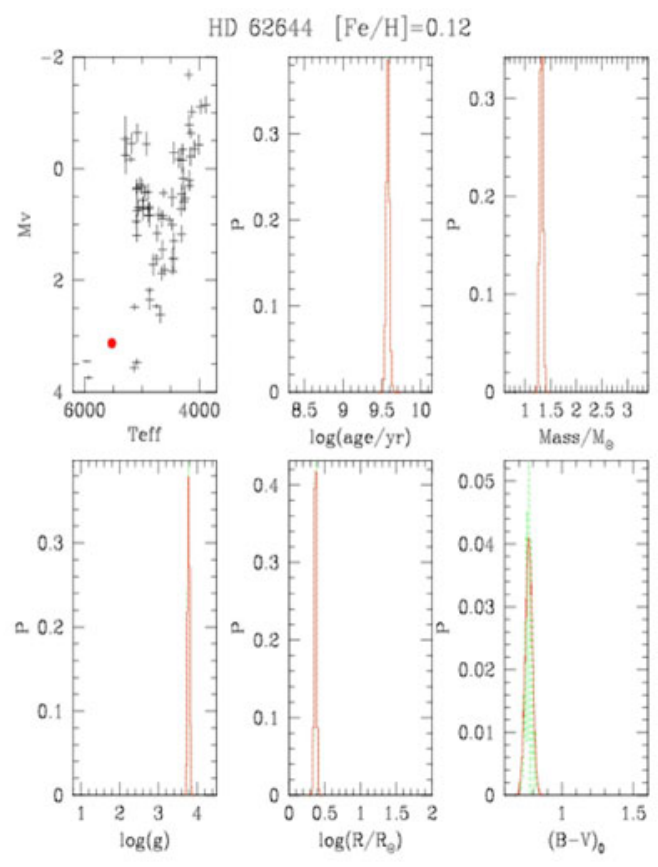

Figure 3. Probability Distribution Functions for one star of our sample. For this object all the PDFs are very well peaked and give unambiguous results (from da Silva et al. 2006).

\section{Observational Results}

The number of giants hosting planets is not large enough to allow refined analysis, but it can start to provide strong indications on several aspects such as planetary frequency, dependence on metallicity, planets characteristics, which can be compared with similar properties in main sequence stars.

\subsection{Radial Velocity Statistics of the Surveys}

After several years of observations, we can summarize the general statistics we have obtained separately for the southern and northern samples. Table 1 summarizes our main findings in terms of RV behavior.

A few aspects of this table require some comments. In the introduction we have mentioned that, when analyzing giant stars, special care should be taken in excluding other sources of RV variability. Indeed out of the $32 \mathrm{RV}$ variable in the Southern hemisphere, 5 stars show associated chromospheric variability and/or bisector variability in phase with the RV period. An example is given in Figure 5, which shows 'planet-like' RV variability that is correlated with Ca II core variations. Therefore these RV variations are likely caused by chromospheric activity rather than due a planetary companion. For the 'binaries' bin we included all stars which showed RV variability of several $\mathrm{km} \mathrm{s}^{-1}$, but for which we have insufficent data to calculate for them a full orbital solutions. For the southern sample they were simply removed from future RV follow-up measurements. For the northern sample we will continue to obtain RV measurements, but with less frequent sampling, in order to derive the orbit.

Clearly, most numbers agree very well between the two surveys. The only real discrepancy is in the number of 'RV constant stars', whose percentage is much larger in the southern sample. However, at issue here is what is defined as a "constant star". The 


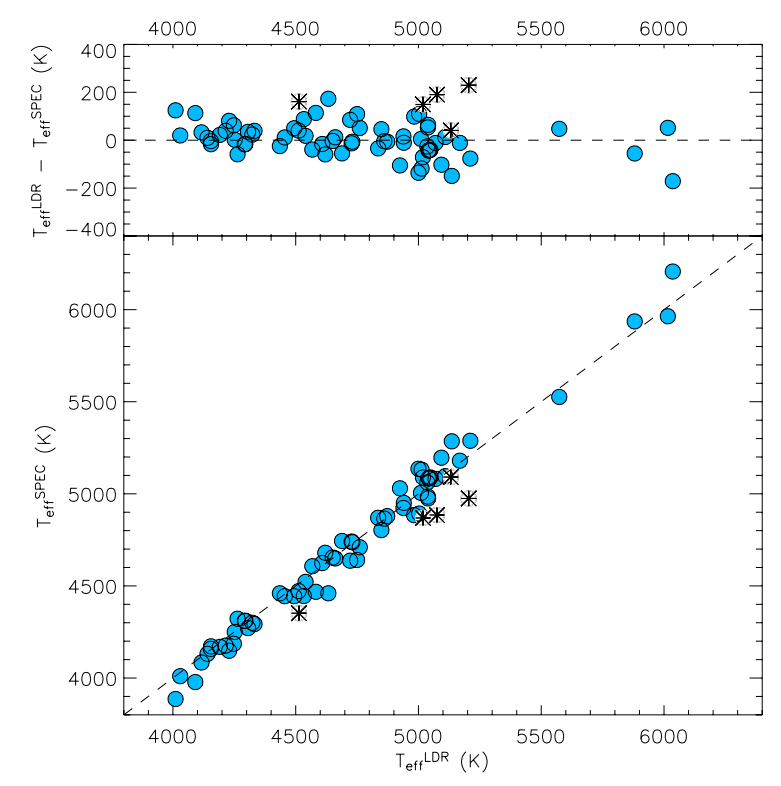

Figure 4. Comparison between effective temperatures obtained from the abundance analysis and effective temperature as obtained by the Line Depth Ratio method for the stars of the southern sample. The agreement is excellent (From Biazzo et al. 2007b)

FEROS program had a measurement precision four times worse than for the northern sample. Not surprisingly, this program revealed more "constant stars". Several of the northern sample giant stars have RV variations of $\approx 20 \mathrm{~m} \mathrm{~s}^{-1}$ which were clearly variable, but would have qualified as a constant star in the southern program. With increased measurement precision many of the constant stars measured by FEROS may turn out to be variable. We therefore confirm the first conclusions obtained by e.g. Walker et al. (1989) that $\mathrm{G}$ and $\mathrm{K}$ giants are a new class of Radial Velocity variable.

The second important result is that giant planets around giant stars seem to be common. Their frequency is of at least $10 \%$, since our findings probably represent a lower limit. We also note that the long orbital periods require observations which extend over a baseline of several years. Continued monitoring may reveal more stars in our sample as hosting giant planets. The planets span a range in periods between 150 and 1000 days, and in mass between 3 and 15 Jupiter masses. We are indeed sensitive only to quite massive planets, given the limited precision of our observations and the fairly high intrinsic RV variability of evolved stars. Thus a $10 \%$ frequency of very massive planets around more massive stars than the sun may represent a minimum value. These results seem to agree well with other surveys around evolved stars, which find massive planets and even a good rate of brown dwarfs orbiting around giant stars (see i.e. Quirrenbach 2008).

Out of the 13 exoplanets so far discovered in our surveys, only 4 have been published (Setiawan et al. 2003a, Setiawan et al. 2004, Setiawan et al. 2005, Döllinger et al. 2007), while the others are in preparation. We show in Figure 6 the RV curves of the 6 planet candidates from the Northern sample. 
Table 1. Overview of the Radial Velocity variability obtained in our surveys.

\begin{tabular}{ccc}
\hline & Northern Sample (76) & Southern Sample (62) \\
\hline Binaries & $15(20 \%)$ & $13(21 \%)$ \\
\hline Variables & $32(43 \%)$ & 22 Long Period $(34 \%)$ \\
& 5 Activity Modulation & 19 Short Period (31\%) \\
\hline Planets & $7(10 \%)$ & $6(10 \%)$ \\
\hline Constant & $21(27 \%)$ & $2(3 \%)$ \\
\hline Precision & $22 \mathrm{~m} / \mathrm{sec}$ & $\sim 5 \mathrm{~m} / \mathrm{sec}$ \\
\hline
\end{tabular}

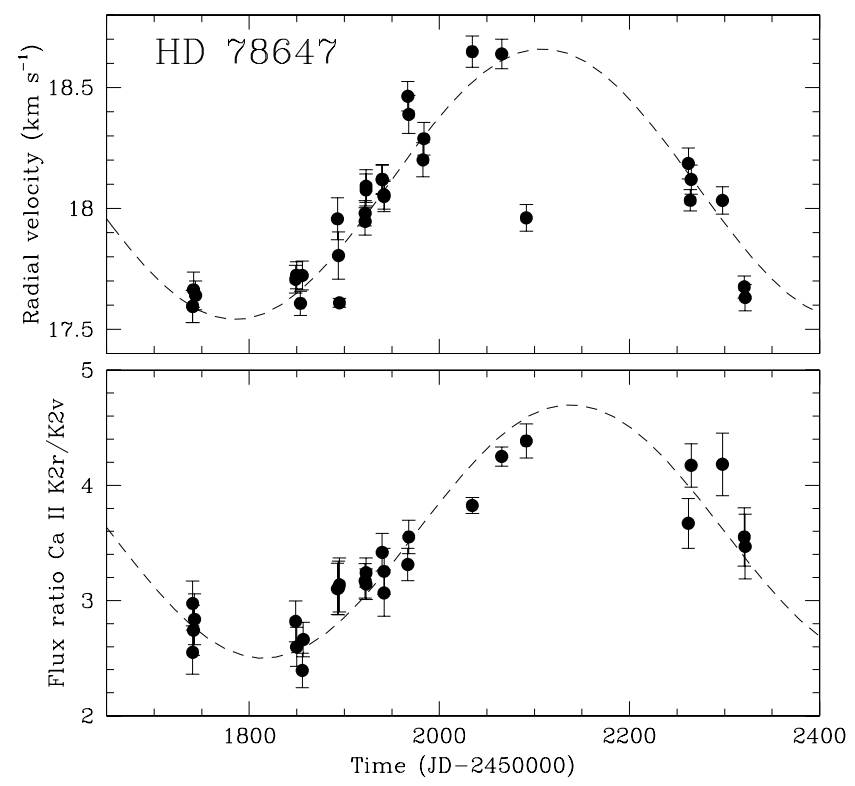

Figure 5. Variations of chromospheric flux ( Ca II K line core ) in phase with the radial velocity period of one giant. These variations indicate that the associate Radial Velocity orbit is not due to the presence of planetary companion, rather by variable chromospheric activity.

\subsection{Stellar Metallicity and Planets}

Gonzales et al. $(1997,2001)$ first showed that stars hosting exoplanets tend to have higher metallicities than stars without giant planets. Subsequent investigations showed this to be a real effect and not an observational bias. (e.g. Santos et al. 2004, 2006, Fisher and Valenti 2005).

The first determinations of abundances of planet-hosting giant stars suggested that such a metallicity-planet connection may not hold for evolved stars (Sadakane et al. 2005 Schuler et al. 2005). This was based, however, on a small sample so no reliable conclusions could be drawn.

Using the first results from our Southern and Northern surveys plus literature data Pasquini et al. (2007) have shown that this suspected trend was correct: giant planethosting stars have the same metallicity distribution as giant stars without planets, and have a different abundance distribution from planet-hosting main sequence stars. Figure 7 

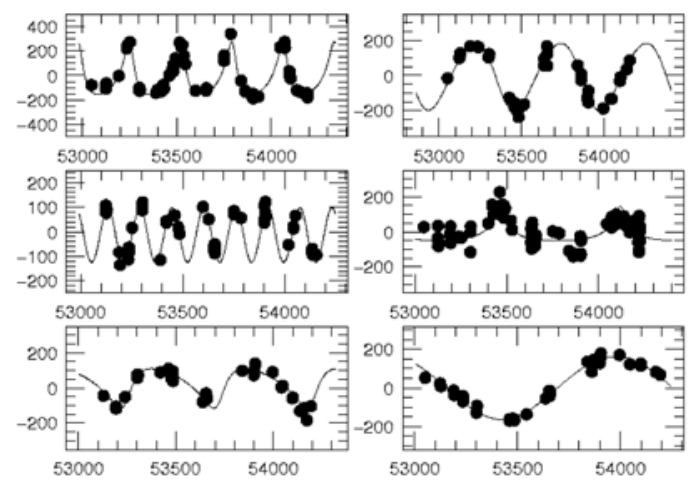

Figure 6. Radial Velocity variations for the 6 stars of the northern sample hosting planets. (Döllinger et al. 2008a,b in preparation)

shows the age-metallicity distribution for the giants of our samples, and for the giants hosting planets (including data from literature), and it is clear that the hosting planets giants do follow the same age-metallicity relationship of the sample stars.

Admittedly, the mixing of stars from literature and our own sample may introduce some bias, because we do not know the parent sample from which the literature data are taken, or if the quoted abundances are on the same scale as the one of our sample. We have therefore computed the metallicity distribution of the planet hosting (13) and non planet-hosting (126) stars of our samples, and this is shown in Figure 8. Clearly the two distributions overlap and there is no indication of metal excess in the planet-hosting stars.

Our result are indirectly supported by the first 5 planets included in the survey of evolved A stars by Johnson et al. ( 2007a), which have a rather uniform metal distribution. We cannot derive more firm conclusions from this sample because we do not know the distribution of the parent sample and being a young population we do expect that its age distribution should be quite metal rich.

For sake of completeness we report that Hekker \& Melendez (2007) claim a possible metal excess in their anaysis of giants hosting planets. We however notice two potential problems in their analysis: the metal excess is entirely due to the presence of two subgiants with exceptionally high metallicity. We have basically no subgiants in our sample. The second and most important problem is that the planet host sample used is not derived by their parent sample: the planet sample is taken from other surveys, whose metal distribution is not known. Clearly this is a flaw, and it is clear from their figures, which show that no star is present in the parent sample in the highest metallicity bins. Without a knowledge of the parent sample from which the planet hosts stars have been derived, a comparison is subject to possible bias and is thus inconclusive.

Concerning metallicity the evidence so far collected seems clear: giant stars hosting planets do not show any tendency towards being metal rich.

\subsection{Planets Characteristics}

The planets found around giant stars have long periods (longer than 150 days), and relatively large masses. In this section we briefly investigate some of the planets' characteristics. 


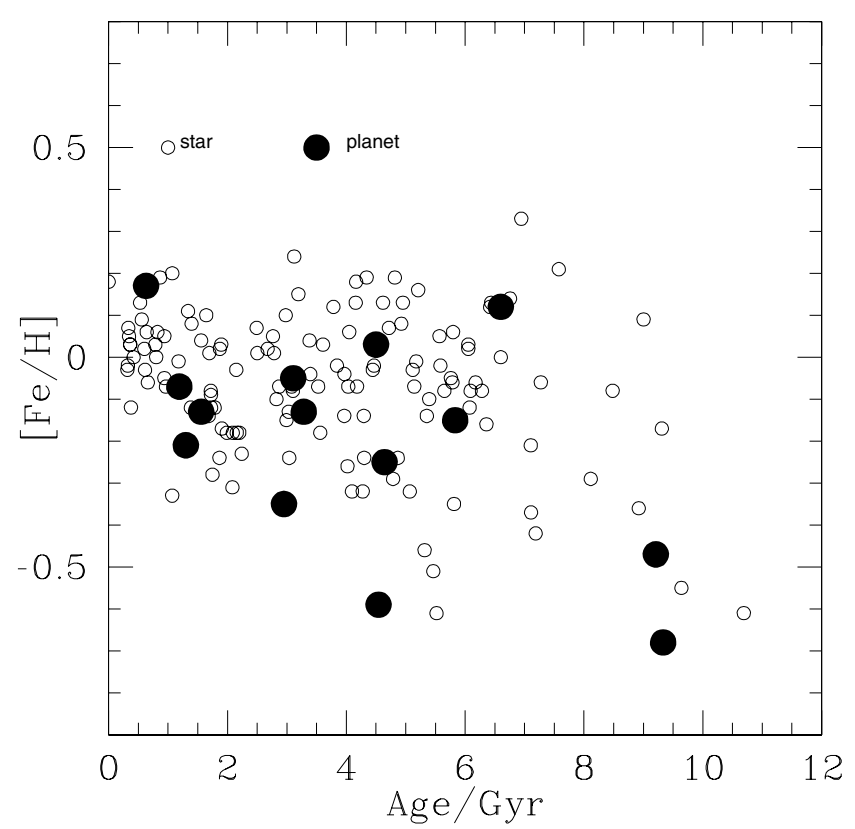

Figure 7. Age-metallicity relationship for the giants of our Southern and Northern samples and for giants with planets, either from our survey or from literature. Clearly there is no difference between the two distributions. (From Pasquini et al. 2007)

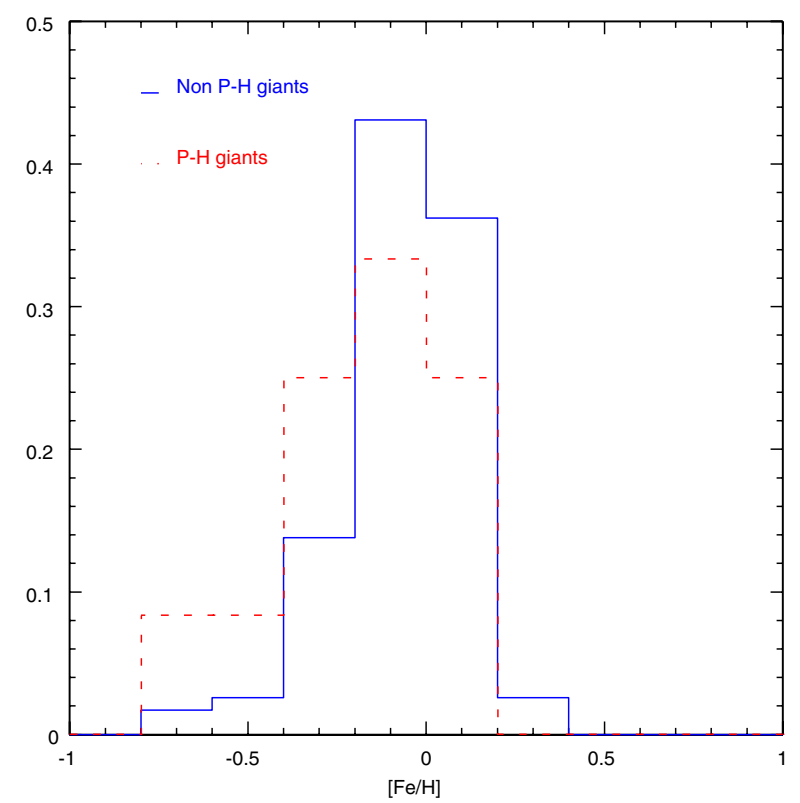

Figure 8. Metallicity distribution of the (126) Southern and Northern giants from our sample not hosting planets (Blue continuous line). With the red dashed line are indicated the 13 giants hosting planets, found in the same samples. No data from literature are included. This comparison clearly shows that hosting planet giants do not prefer metal rich systems. 


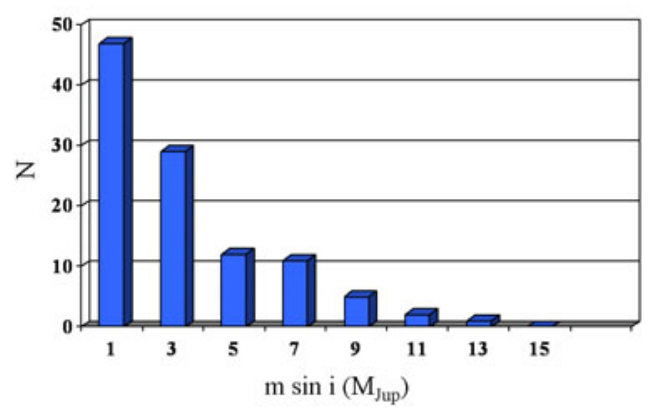

Figure 9. Mass distribution of the planets around stars with masses below $1.1 \mathrm{M}_{\odot}$ showing the strong increase for small planet mass.

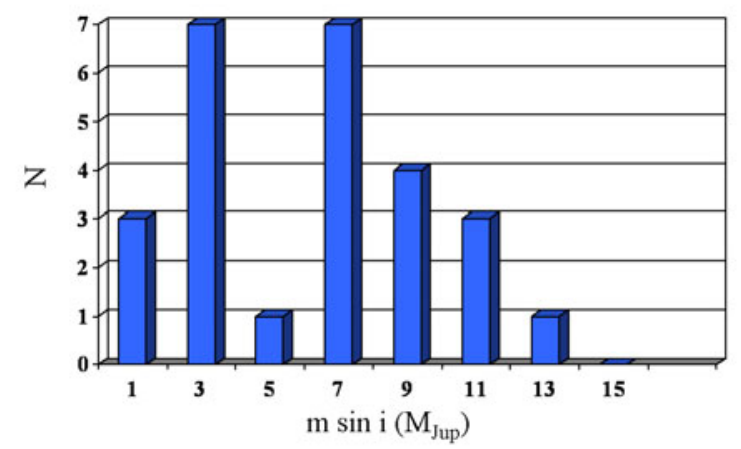

Figure 10. Mass distribution of the planets around stars with masses above $1.1 \mathrm{M}_{\odot}$ (dominated by the giants) and for stars with smaller masses. The distribution is clearly different, with more massive stars showing a very high frequency of massive planets.

Planet masses for giant stars are typically larger than what has been observed around main sequence stars. Figure 9 shows the planet mass distribution for main sequence stars with masses below $1.1 \mathrm{M}_{\odot}$ while Figure 10 is the same but for stars (mostly giants) with masses larger than $1.1 \mathrm{M}_{\odot}$. Clearly the distribution of planet masses for low mass main sequence stars increases towards lower mass planets, and it is definitely different from the one of more massive stars. Of course we know that there are biases in the giants' sample, in that small mass planets are presently out of reach of surveys due to their limited precision and to the intrinsic variability of the stars. Nevertheless, if we assume that the difference between the two distribution is due to observational biases and the distribution was the same than the low mass stars, the plot of Figure 10 would imply an extremely high planet occurrence for the massive stars.

We shall therefore consider as an observational evidence that planets around giants (more massive stars) are more massive than those around lower mass stars. If they were 
sharing a similar overall mass distribution, the frequency so far observed among giants would imply a very high planetary frequency around massive stars.

As far as orbital parameters are concerned, only long period planets are found around giants. This is quite expected since the stars have radii typically as large as $10 R_{\odot}$, therefore short period planets would be swallowed by the stars. It would be interesting to study the case of migration and planetary evolution in giants, in the presence of radius growth and mass losses, as occurring along the RGB and AGB phases. We note that the short period RV variations in giant stars may mask the detection of possible short period planets that may still reside outside the photosphere of the star. These would have orbital periods of many days, or similar to the periods for stellar oscillations. Such variability if found by RV surveys may be dismissed as due to stellar oscillations rather than a short period companion.

The distribution of eccentricity is very similar to what is observed among main sequence stars and ranges from planets in nearly circular orbits to those in highly eccentric orbits. It seems therefore that eccentricity is not affected by the stellar characteristics such as the mass. We note that there could be also here a hidden bias in the eccentricity distribution: orbits with high eccentricity are much more difficult to be mimiced by other phenomena, like oscillations or stellar surface structure so such RV variations are more easily attributed to Keplerian motion. A nearly sinsoidal RV variations can also arise from stellar surface structure and is thus more suspicious. Additional tests (e.g. lack of photometric, Ca II H\&K, or line bisector variations) are needed to confirm these as planetary companions.

\section{Interpretation}

It is natural to interpret the differences between giants' and main sequence stars starting from their three main differences: giants are on average more massive, have larger convective zones and larger radii.

Are giants hosting planets indeed more massive than their main sequence stars counterparts? Given a volume-limited samples this would be a natural result of stellar evolution, and this difference is confirmed in Figure 11, which shows the mass-metallicity distribution for main sequence stars and giants hosting planets. Even if the giants we studied are not really originating from very massive stars, their mass is, on average, higher than that of main sequence stars.

As far as the tendency for planet-hosting giant stars not to be metal rich, we favor the hypothesis that the difference between main sequence and giants is due to pollution: the most external parts of main sequence atmospheres are polluted by engulfed debris and small planets which have a higher metal content. Because of the relatively shallow convection zone of stars on the main sequence, these metals are well mixed only in a small fraction of the star. During the giant phase the convective zone of the stars greatly deepens and the more efficient mixing of the atmosphere dilutes the atmospheric abundance of metals. This scenario has been debated at length in literature, and most analyses conclude against this hypothesis (Santos et al. 2004, 2006, Fischer and Valenti 2005, Ecuvillon et al. 2006). We find that the counter arguments are not particularly strong, mostly because it is not very well known the real depth of the mixing zone in main sequence stars and its variation with stellar mass (cfr. Vauclair 2006). By comparison this effect is greatly enhanced (a factor $\sim 30$ or more) as soon as a star evolves and becomes a red giant. A similar effect should happen at the very low mass end of the stellar mass distribution, where the stars become fully convective. If highly convective $\mathrm{M}$ stars show a non-dependence on metallicity similar to the giants, as seems from the first analysis 


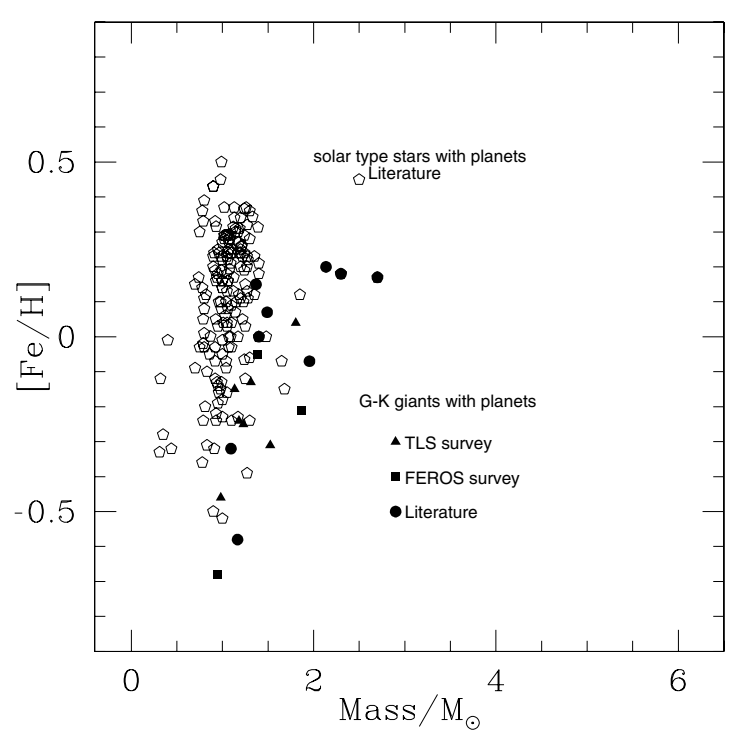

Figure 11. Mass-Metalicity distribution of hosting - planets dwarfs and giants. As expected, evolved stars are on average more massive than main sequence stars.

(Bonfils et al., these proceedings), then the evidence would be even stronger. The main observational argument against this interpretation stems in the subgiants analyzed by Fischer and Valenti (2005), which do not observe any difference between subgiants in the blue and red part of the CMD. Stars on the two sides of the Hertzpung gap (i.e. before and after the deep convective zones have developed) show no differences in metallicity. We can offer no explanation for this point. We already noticed as the metal distribution of subgiants shows a tail at very high metallicity which is not present in the giant samples analyzed so far, and we also note that Murray et al. (2001) reached opposite conclusions than Fischer and Valenti. The issue is still open and we believe that subgiants are worth a dedicated study.

As far the trend that giant stars might have a higher frequency of more massive planets, stellar mass seems the most likely governing parameter, possibly because massive stars may develop more massive proto-planetary disks. Similar conclusions have been reached recently by two papers (Lovis and Mayor 2007, Johnson et al. 2007b), which discussed stars in clusters and in field low mass stars. It is also highly suggestive that while Paulson et al. (2004) did not find any planet around almost 100 low mass stars surveyed in the Hyades, one planet has been found around one of the 2 single giants of this cluster (Sato et al. 2007). As far to this topic, we can observe that all analyses so far agree with an enhanced frequency (and higher mass) of planets around more massive stars. We note also that a similar behavior is present in the samples of Fischer and Valenti (2005) and Santos et al. 2006, but both authors conclude that this dependence was uniquely the result of a selection bias, and that metallicity is the relevant parameter, because metallicity and stellar mass were strongly correlated in their samples. Since giants do not show a dependence of planet formation on metallicity and widen the range of stellar masses observed, we propose that there may be a strong dependence of planet formation on stellar mass. Clearly, more studies are needed to disentangle the possible effects of various stellar parameters (e.g. mass, abundance) on planet formation. 


\section{Giants and Planet Formation Theories}

Considering the 'classical' planet formation schemes: core accretion (Ida \& Lin 2004) and disk instability (Boss 1997), we cannot conclude that the characteristics of the planets around giant stars favor either of the two schemes. It is on the other hand accepted that the metal dependence of massive planet formation in main sequence stars is considered a great success of the core accretion scenario (Ida \& Lin 2004).

It is very possible that reality is more complex than firstly envisaged; could it be that the two mechanisms are both at work, and one is favored with respect to the other depending, for instance, on the stellar mass? Something in this line has been proposed by Matsuo et al. (2007).

On the other hand, a more complex core accretion scenario, which involves the interaction with the stellar magnetosphere and the position of the dust evaporation line for intermediate mass stars, in addition of the snow line considered in less massive stars, could also explain the observations (Lin, private communication).

We do not know yet the answer, but clearly the extension of planet search to giants is bringing new insides, which should be strongly pursued.

\section{Next steps}

In this last section we would like to express our vision for the continuation of this exciting research.

Obviously, the results from other surveys are needed to confirm our results, and to better quantify them. Reaching smaller mass planets would also be essential, to determine the planet mass distribution among intermediate mass stars. The results presented at this conference by other groups are extremely encouraging, and promise to bring a wealth of new results in a few years.

We stress that a proper analysis of the hosting stars is essential, if we aim at obtaining results which can express more about physics. The parent sample require to be analyzed as carefully as the planet hosting stars.

Open clusters, where stars are supposed to share the same age and chemical compositions, could bring direct new results. Similarly subgiants are worth to have a dedicated study. In principle it would be great to compare equivalent samples of field giants and dwarfs, but this seems quite difficult, when all the variables (including age) are considered.

Since ultimately we will aim at comparing stars in different evolutionary status, one important point is to be sure that the analysis methods do not suffer of biases and peculiarities when applied at stars of different evolutionary status. Open clusters could be again privileged testbeds: analyzing stars in a well populated open cluster from main sequence to tip of RGB would be a crucial test to ensure that the results obtained do not suffer of any bias (see e.g. the analysis of Pasquini et al. 2004 of the open cluster IC4651).

\section{Acknowledgements}

The observations have been collected at ESO and the $2 \mathrm{~m}$ Alfred Jensch Telescope of the Thüringer Landessternwarte Tautenburg.

\section{References}

Biazzo, K. et al. 2007a, AN 328, 938

Biazzo, K., Pasquini, L. et al. 2007b, A\&A 475, 981 
Boss, A. P. 1997, Science, 276, 1836

da Silva, L., Girardi, L., et al. 2006, A\&A 458, 603

Döllinger. M. P., et al. 2007a: A\&A, 472, 649

Döllinger. M. P., et al. 2007b: A\&A, in preparation

Döllinger. M. P., et al. 2007c: A\&A, in preparation

Ecuvillon, A., Israelian, G., et al. 2006, A\&A, 449, 809

Fischer, D., \& Valenti, J. 2005, ApJ, 622, 1102

Frandsen, S., Carrier, F., Aerts, C. et al. 2002, A\&A, 394, L5.

Gonzalez, G. 1997, MNRAS, 285, 403

Gonzalez, G. 1998, A\&A, 334, 221

Gonzalez, G., Laws, C. et al. 2001, AJ, 121, 432

Gray, D. Johnes, H. 1991 PASP 103, 409

Hatzes, A. P., Cochran, W. D. 1993, ApJ, 413, 339

Hatzes, A. P., Cochran, W. D. 1994, ApJ, 422, 366

Hekker, Melendez 2007 astro-ph 07091145

Ida, S., \& Lin, D. N. C. 2004, ApJ, 616, 567

Johnson, J. et al. 2007a ApJ 665, 785

Johnson, J. et al. 2007b ArXiv 0707.0518

J $\phi$ rgensen, B.R., Lindegren, L. 2005, A\&A, 436, 127

Laughlin, G., \& Adams, F. C. 1997, ApJ, 491, L51

Lovis, C., Mayor, M. 2007 A\&A 472, 657

Matsuo, T., et al. 2007, ApJ, in press (astro-ph 0703237)

Mayor, M., \& Queloz, D. 1995, Nature, 378, 355

Murray N., Chaboyer B. 2001, ApJ 555, 801

Pasquini, L., Randich, S. et al. 2004, A\&A 424, 951

Pasquini, L., Döllinger, M. Weiss, A. et al. 2007, A\&A 473, 979

Paulson, D.B., Cochran, B., Hatzes, A.P. 2004, AJ 127, 3579

Pollack, J. B., Hubickyj, O., et al. 1996, Icarus, 124, 62

Sadakane, K., Ohnishi, T., Ohkubo, M., Takeda, Y. 2005, PASJ 57, 127

Santos, N. C., Israelian, G., Mayor, M. 2000, A\&A, 363, 228

Santos, N. C., Israelian, G., Mayor, M. 2001, A\&A, 373, 1019

Santos, N. C., Israelian, et al. 2003, A\&A, 398, 363

Santos, N. C., Israelian, G., Mayor, M. 2004, A\&A, 415, 1153

Santos, N. C., Israelian, G., et al. 2005, A\&A, 437, 1127

Sato, B., Ando, H., Kambe, E. 2003, ApJ, 597, L157

Sato, B., Izumiura, H., et al. 2007, ApJ, 661, 527

Schuler, S., Kim, J.H. et al. 2005, ApJ, 632, L131

Setiawan, J., Hatzes, A.P., et al. 2003a, A\&A, 398, L19

Setiawan, J., Pasquini, L., et al. 2003b, A\&A, 397, 1151

Setiawan, J., Pasquini, L., et al. 2004, A\&A 421, 241

Setiawan, J., Rodman, J., et al. 2005, A\&A, 437, L31

Vauclair S. 2004, ApJ 605, 874

Walker, G.A.H., Yang, S., Campbell, B., Irwin, A.W. 1989, ApJ, 343, L21. 